\title{
Closed-Loop Stability Analysis of Voltage Mode Buck Using a Proportional-Delayed Controller
}

\author{
J.-E. Hernández-Díez ${ }^{\mathrm{a}}$, C.-F. Méndez-Barrios*a , S.-I. Niculescu ${ }^{\text {}}$, E.-J. González-Galvána ${ }^{\mathrm{a}}$, G. Mejía-Rodríguez ${ }^{\mathrm{a}}$ \\ \& V. Ramírez-Rivera ${ }^{\mathrm{c}}$
}

\begin{abstract}
This paper focuses on the design of a $P-\delta$ controller for the stabilization of a buck DC/DC converter. The basis of this work is a geometric approach which allows to partition the parameters space into regions with constant number of unstable roots. The main contribution of the paper is that it provides an explicit tool to find $P-\delta$ gains ensuring the stability of the closed-loop system. In addition, the proposed methodology enables the design a non-fragile controller with a desired exponential decay rate $\sigma$. In order to illustrate the effectiveness of the proposed controller, some numerical examples are presented.
\end{abstract}

\section{INTRODUCTION}

The generation, conversion and transmission of electrical energy has raised awareness of the relevance of power electronics in today's applications; among the most popular applications we may consider are those related to renewable energies. This fact has established power electronics as an important subject in electrical and electronics engineering. The basic topologies in electrical conversion systems concerns to AC/DC, AC/AC, DC/AC and DC/DC. This paper considers a buck converter, which due to its remarkable efficiency and simplicity is one of the most popular DC/DC converters in power electronics.

On the other hand, in mechanical, electrical and electronics engineering, control theory is also an important subject with suitable applications in these fields. The automation of industrial processes has established the importance of control theory. Low-order controllers are one of the most widely applied strategies to controlled industrial processes (see, e.g., [1], [2], [3]). This "popularity" can be attributed to their particular distinct features: simplicity and ease of implementation.

Among low-order controllers, those of PID-type are known to be able to cope with uncertainties, disturbances, elimination of steady-state errors and transient response improvement (see, for instance, [4]-[5]). However, as reported in [4], [6], the main drawbacks of PID controllers lies in the tuning of the derivative term, which may amplify

*Corresponding Author.

${ }^{a}$ J.-E. Hernández-Díez, C.-F. Méndez-Barrios and E.-J. González-Gálvan are with the University of San Luis Potosí (UASLP), Dr. Manuel Nava 8, Mexico.

${ }^{b}$ S.-I. Niculescu is with the Laboratoire des Signaux et Systèmes (L2S, UMR CNRS 8506), CNRS-Supélec, 3, rue Joliot Curie, 91192, Gif-surYvette, France.

${ }^{c} \mathrm{~V}$. Ramírez-Rivera is with the Centro de Investigación Científica de Yucatán, A.C.(CICY), Carretera Sierra Papacal-Chuburná Puerto, km. 5, Mexico. high-frequency measurement noise. Moreover, as mentioned in [1], [3], [7], the above arguments advise to avoid the derivative action in most applications. In order to circumvent the above mentioned problem, we can notice that the Euler approximation of the derivative,

$$
y^{\prime}(t) \approx \frac{y(t)-y(t-\varepsilon)}{\varepsilon},
$$

for small $\varepsilon>0$, suggests to replace the derivative action by using delays [8], [9]. Nevertheless, it is well recognized that the presence of a delay in the feedback loop of continuoustime systems is often accompanied with oscillations and instability, bandwidth sensitivity, among others (see, for instance, [10], [11]). However, it is worth mentioning that there exist some situations when the delay may improve the system stability as explained in the classical example [12], [13], where an oscillator is controlled by one delay "block": (gain, delay), with positive gains and small delay values (a detailed analysis of such an approach can be found in [14]).

Inspired by the above observations, the design of loworder controllers with delay as a control parameter have been addressed in several works, for example, [9] (stabilizing chains of integrators by using delays), [15] (multiple delay blocks), [16] (bounded input,single delay), to mention a few. It is worth to notice that this type of controllers add two degrees of tuning (gain, delay) to a closed-loop system analysis.

In this paper, we propose the use of a $P-\delta$ controller for the stabilization of a buck DC/DC converter. This controller consists of a low-order controller with two blocks: proportional + proportional-delayed. The control law for this type of scheme is given as:

$$
u(t):=k_{p} e(t)+k_{\delta} e(t-\tau),
$$

where $k_{p}, k_{\delta}$ and $\tau$ are real parameters and $e(t)$ is the error signal of the control scheme. One of the main advantages to study this type of controller is that it is the first step for a further analysis considering a delayed nature in the control law $\left(\tau_{p}\right)$ due to signal processing, i.e.,

$$
u\left(t-\tau_{p}\right)=k_{p} e\left(t-\tau_{p}\right)+k_{\delta} e\left(t-\left(\tau_{p}+\tau\right)\right) .
$$

The proposed approach in this paper includes a deep analysis of the closed-loop characteristic equation, which considers only a delayed term due to the controller nature. This involves problems such as stability, $\sigma$-stability and 
controller fragility, which are studied individually in the following sections.

The organization of the remaining part of the paper is given as follows: Section II discusses the modelling of a buck DC/DC converter. Section III is the most important contribution of this paper and it concerns to the stability criterion of the closed-loop system in two manners: considering any possible delay value (independent-delay stability) and a fixed delay value. Section IV studies the $\sigma$-stability and fragility problems. Section V shows some illustrative results for the application of the methods developed in this paper and some simulation results. Finally, concluding remarks are addressed.

\section{Preliminary Results}

Figure 1 depicts the classical topology of a Buck DC/DC converter, where $v_{s}$ and $v_{o}$ are the supply and output voltage, respectively. This configuration contains four basic elements: inductor $(L)$, capacitor $(C)$, diode $(D)$ and a controlled switch $(Q)$. Furthermore, a resistive load $R$ is assumed. Then, considering a fixed DC voltage supply $v_{s}$, the main idea is to use the switching pattern of $D$ in order to adjust the output voltage $v_{o}$. The most widely used switching technique is the PWM scheme, which consists of creating a switching pattern of $D$ at a fixed frequency $f$ with an activation period $t_{\text {on }}$ such that the duty cycle of the PWM is given as $U:=f \cdot t_{\text {on }}$.

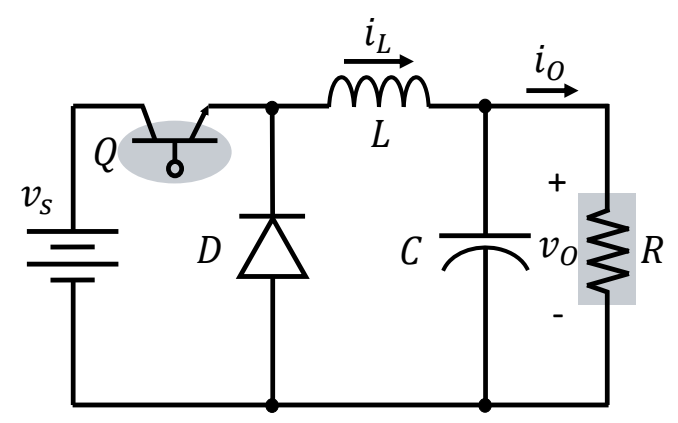

Fig. 1. Topology of the buck DC/DC converter [17].

Motivated by the remarks presented in [17], a linear control formulation can be provided by assuming that all variables have a constant value and a fluctuating part, i.e.,

$$
\begin{aligned}
v_{s}(t) & =V_{s}+\tilde{v}_{s}(t), \\
v_{o}(t) & =V_{o}+\tilde{v}_{o}(t), \\
i_{o}(t) & =i_{o}+\tilde{i}_{o}(t), \\
u(t) & =U+\tilde{u}(t) .
\end{aligned}
$$

Since a resistive load is assumed, then from a control theory perspective, the problem can be formulated as the task to reduce the variations at the output voltage $\tilde{v}_{o}(t)$ despite of possible disturbances in the supply voltage $\tilde{v}_{s}(t)$ and variations in the load by adding a correction factor $\tilde{u}(t)$ to the nominal duty cycle $U$.

The dynamic model of the buck DC/DC converter is derived assuming that the system runs in a continuousconduction mode (CCM). The dynamic model is obtained by defining two operation modes for the switching device
$Q$ : $\mathrm{ON}(\mu=1)$ and $\operatorname{OFF}(\mu=0)$. Applying Kirchoff's law to both equivalent circuits, a switched model is derived. Consequently, by considering a PWM switching pattern, an averaged state-space model can be obtained (see, for instance, [18]), where the averaged states $\left[x_{1}, x_{2}\right]:=\left[i_{L}, v_{o}\right]$ are defined as:

$$
x_{1}:=\frac{1}{T} \int_{t-T}^{t} i_{L}(h) d h, \quad \text { and } \quad x_{2}:=\frac{1}{T} \int_{t-T}^{t} v_{o}(h) d h .
$$

Integrating the switch state $\mu$ over the commutation period $T$, a new control variable $u:=\frac{1}{T} \int_{t-T}^{t} \mu(h) d h$ is defined and it represents the duty cycle. Then, the averaged model of the buck DC/DC converter considering variations in the supply voltage $v_{s}$ is given as:

$$
\begin{aligned}
& \dot{x}_{1}=-\frac{x_{2}}{L}+\frac{V_{s}+\tilde{v}_{s}}{L} u, \\
& \dot{x}_{2}=-\frac{x_{1}}{C}-\frac{x_{2}}{R C} .
\end{aligned}
$$

Remark 1: It is worth mentioning that this averaged model can describe the nature of the system only if the commutation frequency $f$ is sufficiently large.

Now, the relations in the converter for the mean values $\left(V_{s}, V_{o}, I_{o}, U\right)$ can be derived from (7) by setting the derivatives equal to zero, which leads to:

$$
I_{L}=\frac{V_{o}}{R}, \quad V_{o}=U V_{s}
$$

Finally, taking a linear approximation from (7) around the nominal conditions, two transfer functions with respect to the variations in the output voltage $\tilde{v}_{o}$ can be defined as:

$$
\begin{aligned}
G_{1}(s) & :=\frac{\tilde{v}_{o}(s)}{\tilde{u}(s)}=V_{s} \frac{\frac{1}{L C}}{s^{2}+\frac{1}{R C} s+\frac{1}{L C}}, \\
G_{2}(s) & :=\frac{\tilde{v}_{o}(s)}{\tilde{v}_{s}(s)}=U \frac{\frac{1}{L C}}{s^{2}+\frac{1}{R C} s+\frac{1}{L C}} .
\end{aligned}
$$

A linear control approach is considered for ensuring stability in the closed-loop system. More precisely we study the application of the $P-\delta$ controller given by:

$$
C(s):=k_{p}+k_{\delta} e^{-\tau s}
$$

where $k:=\left[k_{p}, k_{\delta}\right]^{T}$ are the controller gains and $\tau$ is a fixed time-delay. We aim to analyze the stability of the system through the closed-loop transfer function:

$$
T(s)=\frac{C(s) G_{1}(s)}{C(s) G_{1}(s)+1} .
$$

\section{Stability AnAlysis}

As established above, we are interested in finding the stability regions in the $\left(k_{p}, k_{\delta}\right)$-parameters space considering a fixed delay-value $\tau$. To this end, let us consider the open-loop transfer function $G_{1}(s)(9)$, along with the $P-\delta$ controller (11), leading to the closed-loop characteristic equation:

$$
\Delta(s):=\frac{L C}{V_{s}} s^{2}+\frac{L}{V_{s} R} s+\frac{1}{V_{s}}+k_{p}+k_{\delta} e^{-\tau s}=0 .
$$


In order to simplify the analysis, in the remaining part of the paper we will adopt the following notation:

$$
a:=\frac{L C}{V_{s}}, \quad b:=\frac{L}{R V_{s}}, \quad c:=\frac{1}{V_{s}} .
$$

In this way, we can rewrite the characteristic equation as:

$$
\Delta(s)=a s^{2}+b s+c+k_{p}+k_{\delta} e^{-\tau s}=0 .
$$

It is well known that the stability of the closed-loop system is directly related to the location of the roots of (15) (see, [11], for further details). More precisely, the closedloop system is stable if and only if all the roots of the characteristic equation are located in the LHP (Left-Half Plane) of the complex plane.

Definition 1 (Frequency crossing set): The frequency crossing set $\Omega \subset \mathbb{R}$ is the set of all $\omega$ such that, there exist at least a triplet $\left(k_{p}, k_{\delta}, \tau\right)$ for which:

$$
\Delta\left(j \omega ; k_{p}, k_{\delta}, \tau\right)=0 .
$$

Remark 2: It is clear that if we take the complex conjugate of (16), the following equality holds:

$$
\Delta\left(-j \omega ; k_{p}, k_{\delta}, \tau\right)=\overline{\Delta\left(j \omega ; k_{p}, k_{\delta}, \tau\right)} .
$$

Therefore, in the rest of the paper we will consider only nonnegative frequencies, i.e., $\Omega \subset \mathbb{R}_{+} \cup\{0\}$.

Definition 2 (Stability Crossing Curves): The stability crossing curves $\mathscr{T}$ is the set of all parameters $\left(k_{p}, k_{\delta}, \tau\right) \in \mathbb{R}^{2} \times \mathbb{R}_{+}$for which there exist at least one $\omega \in \mathbb{R}_{+} \cup\{0\}$ such that $\Delta\left(j \omega ; k_{p}, k_{\delta}, \tau\right)=0$. For a fixed delay value $\tau^{*} \in \mathbb{R}_{+}$, any point $k \in \mathscr{T}$ is known as a crossing point.

\section{A. Delay-Independent Stability}

As mentioned in the introduction, first, we analyze the stability of the closed-loop system considering any possible fixed delay value $\tau \in \mathbb{R}_{+}$, this particular result is shown as follows:

Proposition 1: Let $a, b, c \in \mathbb{R}_{+}$, then, the buck DC/DC converter is asymptotically stable independent of the delay value $\tau \in \mathbb{R}_{+}$, if the controller gains satisfy the following conditions:

$$
\begin{aligned}
k_{\delta}^{2} & <\frac{b^{2}}{4 a^{2}}\left(4 a k_{p}+4 a c-b^{2}\right), \\
k_{p} & >\frac{b^{2}}{4 a}-c .
\end{aligned}
$$

\section{B. Stability Crossing Curves Characterization}

Now, in order to perform a stability analysis for a specific delay value in the controller design, we first need to construct the stability crossing boundaries. Then, it will be useful to characterize the behavior of the roots movement as a parameter variation crosses some of these boundaries. This section focuses on analyzing such a behavior.

Proposition 2: Let $\tau \in \mathbb{R}_{+}$be a fixed value and $\sigma, \omega \in$ $\mathbb{R}$. Then, $\Delta(s)$ has a root at $s=\sigma+j \omega$, if and only if the controller gains $k(\sigma, \omega):=\left[k_{p}, k_{\delta}\right]^{T}$, are given as:

$$
\left\{\begin{array}{l}
k_{p}(\sigma, \omega)=a\left(\omega^{2}-\sigma\right)-(2 a \sigma+b) \omega \cot (\tau \omega)-b \sigma-c, \\
k_{\delta}(\sigma, \omega)=(2 a \sigma+b) \omega e^{\tau \sigma} \csc (\tau \omega), \text { if } \omega \neq 0
\end{array}\right.
$$

$$
k_{\delta}=e^{\tau \sigma}\left[-k_{p}-\left(a \sigma^{2}+b \sigma+c\right)\right] \text {, if } \omega=0 .
$$

The stability crossing curves are characterized in the following result.

Proposition 3: Let $\tau \in \mathbb{R}_{+}$be a fixed delay value and $\Omega:=$ $\bigcup \Omega_{\ell} \bigcup\{0\}$ for $\ell \in \mathbb{N}$, where the subsets $\Omega_{\ell}$ are defined as:

$$
\Omega_{\ell}:=\left\{\omega \in \mathbb{R}_{+} \mid \omega \in\left(\frac{\pi}{\tau}(\ell-1), \frac{\pi}{\tau} \ell\right)\right\} .
$$

Then, $\omega \in \Omega \backslash\{0\}$ is a crossing frequency if and only if $k(\omega):=\left[k_{p}(\omega), k_{\delta}(\omega)\right]^{T}$, where:

$$
\begin{aligned}
& k_{p}(\omega)=a \omega^{2}-c-b \omega \cot (\tau \omega), \\
& k_{\delta}(\omega)=b \omega \csc (\tau \omega),
\end{aligned}
$$

defines a crossing point $k(\omega) \in \mathscr{T}$. Moreover, the line

$$
k_{\delta}=-k_{p}-c
$$

defines a stability crossing curve at $\omega=0$.

Given all stability crossing points $k(\omega)$ and the frequency crossing set $\Omega$, we can define each stability crossing curve through its continuity as follows:

$$
\begin{aligned}
\mathscr{T}_{0} & :=\left\{k \in \mathbb{R}^{2} \mid k_{\delta}=-k_{p}-c\right\}, \\
\mathscr{T}_{\ell} & :=\left\{k(\omega) \in \mathbb{R}^{2} \mid \omega \in \Omega_{\ell} \text { for } \ell \in \mathbb{N}\right\} .
\end{aligned}
$$

Finally, we describe the set $\mathscr{T}$ as:

$$
\mathscr{T}=\bigcup_{\ell} \mathscr{T}_{\ell}, \quad \ell \in \mathbb{N} \cup\{0\} .
$$

\section{Stability Region with a Fixed Delay}

It is clear that if $k_{\delta}(\omega) \neq 0$ for $\omega \in \Omega_{\ell}$ and $\ell \in \mathbb{N}$, then the stability crossing curves $\mathscr{T}_{\ell}$ does not crosses the $k_{p}$-axis. Furthermore, the only curve that crosses the $k_{p}$-axis, precisely at $k_{p}=0$ is $\mathscr{T}_{0}$, which is the line defined in (24). Now, notice that since the physical parameters $L, C$ and $R$ are positive, then $a, b, c \in \mathbb{R}_{+}$. Now, observing the sign of $k_{\delta}(\omega)$, we can conclude that a stability crossing curve $\mathscr{T}_{\ell}$ with $\ell$ even or $\ell$ odd is located above or below the $k_{p}$-axis, respectively. Finally, bearing in mind the above facts, it will be useful to introduce the following sets:

$$
\mathscr{B}_{\ell}:=\left\{\begin{array}{lll}
\tilde{k} \in \mathbb{R}^{2} & \begin{array}{lll}
\tilde{k}_{\delta}<k_{\delta} ; \quad \forall k \in \mathscr{T}_{i} & \text { for } \ell \in 2 \mathbb{N} \\
\widetilde{k}_{\delta}>k_{\delta} ; \quad \forall k \in \mathscr{T}_{\ell} & \text { for } \ell \in 2 \mathbb{N}+1 \\
\widetilde{k}_{\delta}<-\widetilde{k}_{p}-c & \text { for } \ell=0
\end{array} .
\end{array}\right.
$$

Hence, the sets $\mathscr{B}_{\ell}$ are the collection of all points below and above the curves $\mathscr{T}_{\ell}$ for $\ell$ odd and $\ell$ even, respectively, and the set $\mathscr{B}_{0}$ is the set of all points below the stability crossing curve $\mathscr{T}_{0}$. Finally, we have the following proposition:

Proposition 4: Given a fixed delay $\tau \in \mathbb{R}_{+}$, there always exists an open connected stability region $\mathscr{H}$ defined by:

$$
\mathscr{H}:=\bigcap_{\ell \in \mathbb{N}} \mathscr{B}_{\ell} \bigcap \mathscr{B}_{0}
$$

Furthermore, $\mathscr{H}$ is unbounded. 


\section{Crossing Directions Characterization}

In order to compute a stability index which is the number of roots in the RHP for a given parametrical region it is of interest to determine the roots tendency as the vector $k$ deviates from the curve $\mathscr{T}$. The following results are the main tools applied in this paper to achieve such a task.

Proposition 5: A pair of roots of the characteristic equation (15) moves from the LHP to the RHP as $k$ crosses a stability crossing curve $k(\omega)$ with $\omega \neq 0$ in the increasing direction of $k_{\chi}$ for $\chi \in\{p, \delta\}$ if:

$$
C_{\chi} \triangleq b(\tau \omega \cot (\tau \omega)-1) \cos \left(\eta_{\chi} \tau \omega\right)+\eta_{\chi}(b \tau+2 a) \omega \sin (\tau \omega)>0 \text {, }
$$

where the indicative function $\eta_{\chi}$ is defined as:

$$
\eta_{\chi}:=\left\{\begin{array}{cl}
0, & \text { if } \quad \chi=p \\
1, & \text { if } \quad \chi=\delta
\end{array}\right.
$$

Furthermore, the crossing is from the RHP to the LHP if the inequality is reversed.

Observe that Proposition 11 does not give any information about the crossing when $\omega=0$. The following result fills this gap.

Proposition 6: Given a fixed delay $\tau \in \mathbb{R}_{+}$. Then, one root of (15) crosses from the LHP to the RHP of the complex plane through the origin as $k$ crosses $\mathscr{T}_{0}$ from left to right if the intersection of $k$ and $\mathscr{T}_{0}$ is located at the left of the point $k_{0} \in \mathscr{T}_{0}$, defined by:

$$
k_{0}:=\left[k_{p_{0}}, k_{\delta_{0}}\right]^{T}=\frac{1}{\tau}[-\tau b-c, b]^{T} .
$$

Furthermore, the crossing of the root is from the RHP to the LHP if the intersection is located at the right of $k_{0}$.

\section{IV. $\sigma$-STABILITY AND FRAGILITY}

In this section we propose two auxiliary results to deal with the problems of $\sigma$-stability and fragility of a given controller. These results will be useful in the design of a controller that satisfies a given performance (exponential decay rate) as well as some robustness against parametrical uncertainties. To this end, let us first state the $\sigma$-stability problem: Let $\sigma \in \mathbb{R}_{-}$, the $\sigma$-stability problem can be described as the task of determining a controller $k$ such that the real part of the rightmost roots of the characteristic equation (15) is located at the left of $\sigma$. Let $\mathscr{T}_{\sigma}$ denote the set of all $k$ such that (15) has at least one root on the vertical line of the complex plane defined as $L_{\sigma}:=\sigma+j \omega$ for all $\omega \in \mathbb{R}$. This vertical line is defined as the $\sigma$-axis. In order to introduce similar results to those presented in Section III-B we have the following:

Corollary 1: Let $\omega \in \Omega$, and let $\tau \in \mathbb{R}_{+}, \sigma \in \mathbb{R}_{-}$be fixed values. Then, the set $\mathscr{T}_{\sigma}$ can be computed as:

$$
\mathscr{T}_{\sigma}=\mathscr{T}_{\bar{\sigma}} \cup \mathscr{T}_{\sigma, 0},
$$

with

$$
\begin{aligned}
& \mathscr{T}_{\sigma, 0}=\left\{k \in \mathbb{R}^{2} \mid k=k(\sigma, 0)\right\}, \\
& \mathscr{T}_{\bar{\sigma}}=\left\{k \in \mathbb{R}^{2} \mid k=k(\sigma, \omega)\right\} .
\end{aligned}
$$

Consider now the fragility problem, which consists of computing the maximum controller parameters deviation $d$ of a given stabilizing controller $\bar{k}:=\left[\bar{k}_{p}, \bar{k}_{\delta}\right]^{T}$, such that the closed-loop system remains stable, as long as the controller parameters $k$ satisfy the inequality:

$$
\sqrt{\left(k_{p}-\bar{k}_{p}\right)^{2}+\left(k_{\delta}-\bar{k}_{\delta}\right)^{2}}<d .
$$

In order to address this problem, let $k(\omega)=\left[k_{p}(\omega), k_{\delta}(\omega)\right]^{T}$ as given in Proposition 3. Bearing in mind this notation, we have the following:

Proposition 7: Let $\bar{k}$ be a stabilizing controller. Then, the maximum parameter deviation $d$ of $\bar{k}$, such that the closedloop system remains stable, is given by:

$$
d:=\min \left\{\tilde{d}, \frac{1}{\sqrt{2}}\left|\bar{k}_{p}+\bar{k}_{\delta}+c\right|\right\},
$$

with $\tilde{d}$ defined as:

$$
\tilde{d}:=\min _{\omega \in \Omega_{f}}\{\xi(\omega)\},
$$

where $\Omega_{f}$ denote the set of all roots of $f(\omega)$ :

$$
\begin{gathered}
f(\omega):=\left\langle k(\omega)-\bar{k}, \frac{d}{d \omega} k(\omega)\right\rangle . \\
\text { V. ILLUSTRATIVE RESULTS }
\end{gathered}
$$

All results of this section were obtained by means of the "SimPowerSystems" toolbox in the "Simulink" environment of the software "Matlab". The parameters used in the simulation are summarized in Table I. The tests presented in this section are designed to regulate the output voltage $v_{o}(t)$ to a nominal value of $V_{o}:=20$. Recall that the control scheme has the task to regulate the variations of the output voltage $\tilde{v}_{o}(t)$ to zero in order to satisfy the following: $v_{o}(t) \rightarrow V_{o}$. The control law proposed for the achievement of this objectives is given by:

$$
u(t)=U+\tilde{u}(t)
$$

with:

$$
\tilde{u}(t):=k_{p} e(t)+k_{\delta} e(t-\tau),
$$

where the error signal is defined as:

$$
e(t):=0-\tilde{v}_{o}(t)=V_{o}-v_{o}(t),
$$

and the nominal value $U$ can be obtained directly from (8). Consider the controller's delay value $\tau=5 \times 10^{-5} s$ for the

TABLE I

PARAMETERS OF THE SYSTEM

\begin{tabular}{ccc}
\hline \hline Symbol & Value & Unit \\
\hline \hline$R$ & 3 & $\Omega$ \\
$L$ & $180 \times 10^{-5}$ & $H$ \\
$C$ & $40 \times 10^{-6}$ & $F$ \\
$V_{S}$ & 40 & $V$ \\
$f$ & $20 \times 10^{3}$ & $H z$ \\
\hline \hline
\end{tabular}

$P-\delta$ controller (11) along with the parameters given in Table I. Figure 2, first depicts the delay-independent stability region 
obtained directly from Proposition 1 . Second, it illustrates the stability region for the given fixed value $\tau$ obtained by means of Proposition 4 and computed by applying Proposition 3. Making use of these results, the $P-\delta$ controller is set as

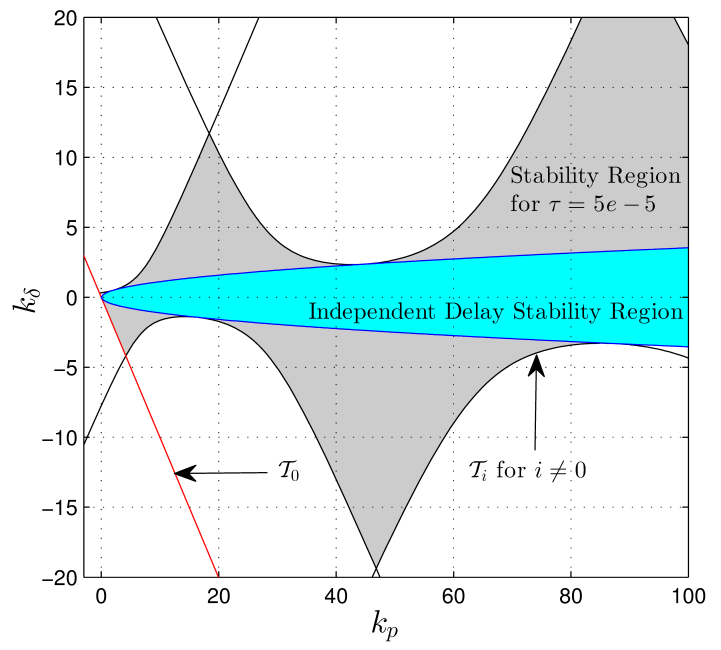

Fig. 2. Stability Region for $\tau=5 \times 10^{-5} s$ and Delay-Independent Stability Region.

$k=[50,1]^{T}$. Three different values of $\tau$ are proposed in order to verify the independent stability condition. The results of the regulation of $v_{o}(t)$ for this test are depicted in Fig. 3.
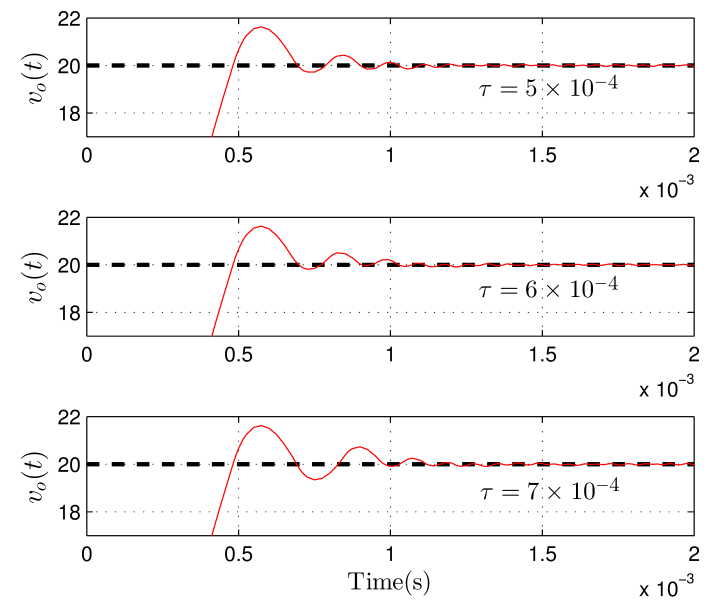

Fig. 3. Closed-Loop System Response Under Different Values of $\tau$.

In order to show how the stability region for the fixed value $\tau=5 \times 10^{-5}$ behaves, a large view of this region is depicted in Fig. 4.

In the following, let us consider the stability index $\eta$ (number of roots in the RHP) for different regions delimited by the stability crossing boundaries. To this end, Fig. 5 presents the results of applying Propositions 5 and 6, where the colors "red", "green", "blue" and "black" stands for $C_{p}>0, C_{\delta}>0, C_{p} C_{\delta}>0$ and $\left(C_{p}<0\right) \&\left(C_{\delta}<0\right)=$ 'true', respectively.

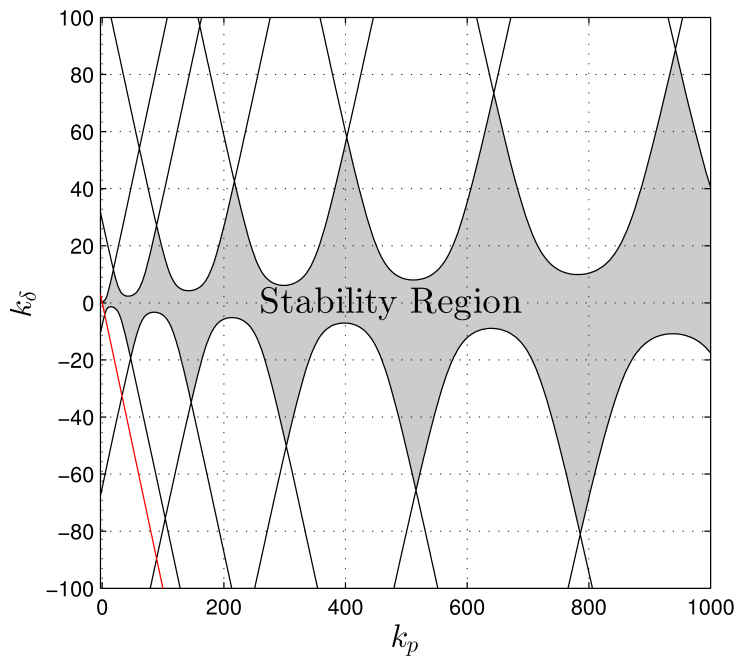

Fig. 4. Stability Region for $\tau=5 \times 10^{-5}$.

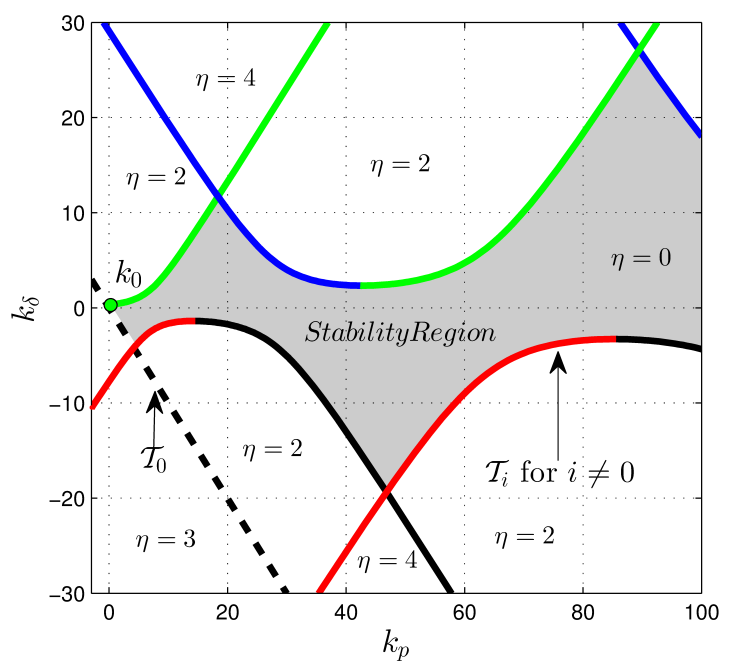

Fig. 5. Stability Index Analysis.

Finally, we apply the auxiliary results shown in Section IV. First we use a $\sigma$-Stability Analysis varying $\sigma$ from 0 to $-4 \times 10^{3}$, the results are summarized in Fig. 6 . From this figure, we denote the finding of a stability region that ensures that all roots of the characteristic equation of the closed-loop system has real part $\sigma<-4 \times 10^{3}$. In other words, that the exponential decay rate of the closed-loop system response is smaller than $4 \times 10^{3}$. Second, we test the fragility for three different controllers, the results are summarized in Table II and illustrated in Fig. 7.

TABLE II

FRAGILITY ANALYSIS

\begin{tabular}{ccccc}
\hline \hline$k$ & $\Omega_{f}$ & $d_{o}$ & $d_{f}$ & $d$ \\
\hline$k_{1}$ & $\{38110,63023,252843\}$ & 1.1431 & 1.6192 & 1.4319 \\
$k_{2}$ & $\{60765,251703,314353\}$ & 15.574 & 3.7049 & 3.7049 \\
$k_{3}$ & $\{120762,253594,31548\}$ & 23.3522 & 5.1322 & 5.1322 \\
\hline
\end{tabular}




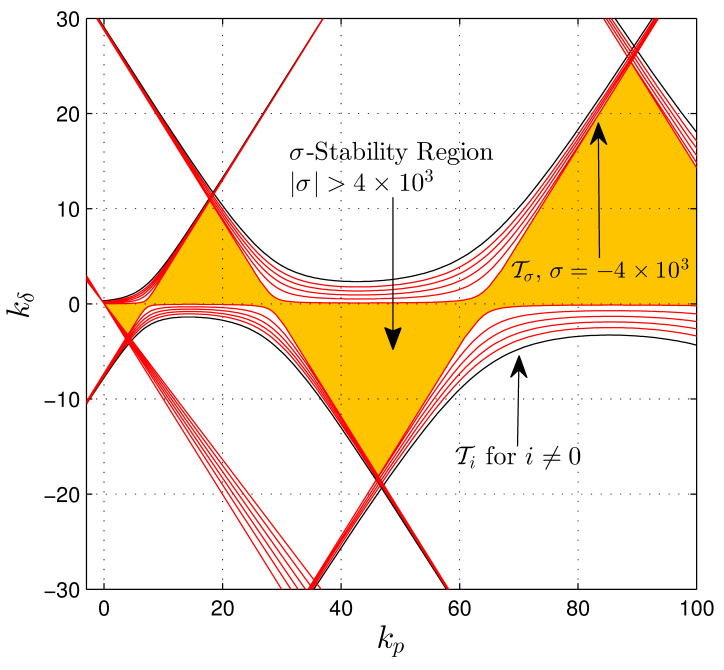

Fig. 6. $\sigma$-Stability Region.

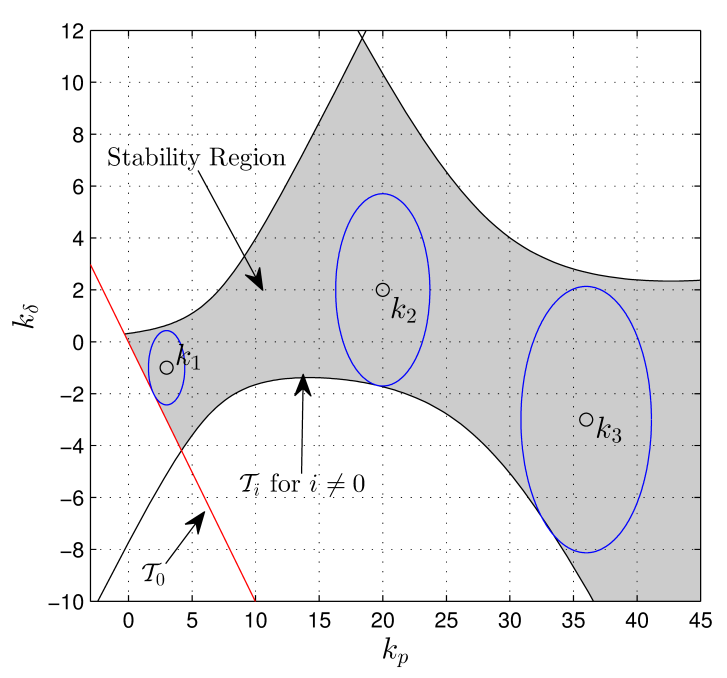

Fig. 7. Fragility Analysis.

\section{CONCLUDING REMARKS}

A methodology for the design of a $P$ - $\delta$ controller applied to the stabilization of a buck DC/DC converter is presented. In addition, the behavior of the roots of the characteristic equation, as the controller gains are varied is analyzed. The results go beyond the stabilization problem, particularly, the closed-loop performance analysis via the solution to the $\sigma$ stability problem and the study of controller fragility are addressed. The design methodology can be applied and developed straightforwardly, showing that the presented results are easy to implement.

\section{REFERENCES}

[1] K. J. Aström and T. Hägglund, "The Future of PID Control," Chem. Eng. Progress, vol. 9, no. 11, pp. 1163-1175, 2001.

[2] G. Silva, A. Datta, and S. Bhattacharrya, PID Controllers for Time Delay Systems, ser. Control Engineering. Boston: Birkhäuser, 2005.

[3] A. O'Dwyer, Handbook of PI and PID Controller Tuning Rules, 3rd ed. London: Imperial College Press (ICP), 2009.

[4] K. J. Aström and T. Hägglund, PID Controllers: Theory, Design, and Tuning, 2nd ed. Instrument Society of America, Research Triangle Park, NC, 1995.

[5] A. Ramírez, S. Mondié, R. Garrido, and R. Sipahi, "Design of Proportional-Integral-Retarded (PIR) Controllers for Second-Order LTI Systems," IEEE Transactions On Automatic Control, vol. 61, no. 6, pp. 1688-1693, 2016.

[6] W. Altmann and D. Macdonald, Practical Process Control for Engineers and Technicians. Elsevier/Newnes, 2005.

[7] J. F. Smuts, Process Control for Practitioners. OptiControls Inc., 2011.

[8] H. Suh and Z. Bien, "Use of Time-Delay Actions in the Controller Design," IEEE Transactions On Automatic Control, vol. 25, no. 3, pp. 600-603, 1980.

[9] S.-I. Niculescu and W. Michiels, "Stabilizing a chain of integrators using multiple delays," IEEE Trans. Aut. Control, vol. 49, no. 5, pp. 802-807, 2004.

[10] S. I. Niculescu, Delay Effects on Stability: A Robust Control Approach, ser. Lecture Notes in Control and Information Sciences. Heidelberg: Springer, 2001.

[11] W. Michiels and S.-I. Niculescu, Stability, Control, and Computation for Time-Delay Systems. An Eigenvalue-Based Approach, ser. Advances in Design and Control. Philadelphia: SIAM, 2014.

[12] C. Abdallah, P. Dorato, J. Benitez-Read, and R. Byrne, "Delayed positive feedback can stabilize oscillatory systems," in Proc. American Contr. Conf., 1993, pp. 3106-3107.

[13] R. Sipahi, S. I. Niculescu, C. Abdallah, T. Chaouki, W. Michiels, and K. Gu, "Stability and Stabilization of Systems with Time Delay: Limitations and Opportunities," IEEE Control Systems Magazine, vol. 31, no. 1, pp. 38-65, 2011.

[14] S.-I. Niculescu, W. Michiels, K. Gu, and C. Abdallah, "Delay effects on output feedback control of dynamical systems," in Complex Time Delay Systems, F. Atay, Ed. Berlin: Springer-Verlag, 2010, pp. 63-84.

[15] V. Kharitonov, S.-I. Niculescu, J. Moreno, and W. Michiels, "Static output feedback stabilization: Necessary conditions for multiple delay controllers," IEEE Transactions On Automatic Control, vol. 50, no. 1, pp. 82-86, 2005.

[16] F. Mazenc, S. Mondié, and S.-I. Niculescu, "Global asymptotic stabilization for chains of integrators with a delay in the input," IEEE Transactions on Automatic Control, vol. 48, no. 1, pp. 57-63, 2003.

[17] D. U. Campos-Delgado and D. R. Espinoza-Trejo, "Educational experiments in power electronics and control theory: D.c. switched power supplies," International Journal of Electrical Engineering Education, 2000.

[18] M. H. Rashid, Power Electronics: Circuits, Devices and Applications. Prentice Hall, 2003. 